\title{
La experiencia femenina y su vinculación con el territorio en el poemario Mujeres a la intemperie/ Pu zomo wekentu mew de Liliana Ancalao
}

\author{
The female experience and its connection with the territory in the poems \\ Mujeres a la intemperie/ Pu zomo wekentu mew by Liliana Ancalao
}

\author{
Elvira Rodríguez ${ }^{2}$
}

\begin{abstract}
Resumen
Con la instalación del Estado argentino en el territorio mapuche se hizo una borradura de las comunidades existentes, homogenizando las prácticas sociales y culturales a partir del proyecto nacional. En ese escenario, la poesía mapuche del Puelmapu permite poner en tensión los discursos oficiales, problematizando los discursos monoculturales y cuestionando la situación cultural, social y territorial. La poesía recoge la experiencia de las historias borradas o faltantes, las migraciones y la incorporación al mundo laboral en la ciudad. En ese contexto, surge la poesía de Liliana Ancalao, profesora y activista cultural, perteneciente a la comunidad mapuche-tehuelche Ñamkulawen. El objetivo del presente artículo es constatar cómo en el poemario Mujeres a la intemperie/ Pu zomo wekuntu mew, reeditado como Resuello/ Neyen se establece un vínculo entre la experiencia femenina y la recuperación del territorio. En los poemas seleccionados se desarrolla una relación directa entre el cuerpo y el lugar, dejando de manifiesto los distintos procesos históricos que se van grabando en su poesía. A través de la escritura, se logra restablecer los lazos con el lugar, con la memoria y la identidad, visibilizando la experiencia colectiva de las mujeres que se encuentran a la intemperie, olvidadas por los relatos maestros.
\end{abstract}

Palabras clave: poesía, cuerpo, lugar, territorio, Liliana Ancalao.

\begin{abstract}
When the Argentinian government settled in the Mapuche territory, many existing communities ceased to exist. This caused the homogenization of social and cultural practices as part of the national project. In this scenario, the Puelmapu's mapuche poetry set the official discourse in tension by questioning monocultural discourses and the cultural, social and territorial situation. This poetry collects experiences from the erased or missing stories, the migrations, and the assimilation into the urban working world. The poetry of Liliana Ancalao emerges in this context, as the voice of a teacher and cultural activist who belongs to the Mapuche-
\end{abstract}

\footnotetext{
${ }^{1}$ Este artículo fue parte de la tesis La poesía mapuche y su vinculación con los movimientos sociales. Caso chileno y argentino para optar al grado de Doctora en Estudios Americano, especialidad en Pensamiento y Cultura, IDEA/ USACh. Estos estudios fueron financiados gracias a Beca Conicyt - Doctorado Nacional, año 2016, $\mathrm{n}^{\circ}$ de folio 21160879.

${ }^{2}$ Escuela de ingeniería en Medio Ambiente y Sustentabilidad. Universidad Mayor, Chile. Correo: elvira.rodriguez@umayor.cl. ORCID ID: https://orcid.org/0000-0002-9527-7568?lang=es
}

Recibido: 30.04 .2021

Aceptado: 10.07.2021 


\section{Elvira Rodríguez}

Tehuelche community, Namkulawen. The aim of this article is to assert how the collection of poems Mujeres a la intemperie/ Pu zomo wekuntu mew (Women out in the open), republished as Resuello/ Neyen (Gasp), establishes a close link between the female experience and land recovery. The chosen poems evidence a direct relationship between body and place, unfolding several historical processes inscribed in her poetry. Through her writing, we can restore ties with the territory, memory, and identity by raising the visibility of the collective experience of women out in the open, forgotten by the master narratives.

Key words: poetry, body, place, territory, Liliana Ancalao.

El mameluco de mi viejo espera jubilarse por el resfrío crónico del pozo

y el dolor de las espaldas

\section{Introducción}

La poesía mapuche ha logrado constituirse como parte del canon regional, articulando discursos multiculturales y cosmopolitas, permitiendo recuperar una voz, una historia y una tradición. Para las nuevas generaciones ha sido una herramienta para visibilizar sus demandas, pero por sobre todas las cosas, una forma de expresión literaria que se viene publicando desde 1917. En aquella multiplicidad de voces encontramos la obra de la poeta argentina Liliana Ancalao (1961) profesora y activista cultural, proveniente de Comodoro Rivadavia, perteneciente a la comunidad mapuchetehuelche Namkulawen.

La obra de Liliana Ancalao -Resuello, Tejidos con lana cruda y Mujeres a la intemperiePu zomo wekuntu mew-, ha sido trabajada a partir de la autotraducción y cómo en su obra se desarrollan discursos que apuntan la reapropiación y descolonización (Stocco, 2017), el postmo nolingüismo y la resistencia cultural (Stocco, 2018) y el tránsito entre la oralidad, escritura y traducción (Mellado, 2014). Su poesía, pionera como tal en el territorio del Puelmapu, no se reconoce desde el comienzo como poesía mapuche, sino como "literatura patagónica" (Moraga, 2010). Sus primeros textos circulan como cartillas artesanales que se entregaban de manera gratuita en las calles. Su escritura, como señala Moraga (2010) "intentaba dar cuenta de una sujeto tensionado entre un desarraigo urbano y una cultura mapuche palpitante" (p. 133). Destacamos la idea del desarraigo, pues la escritura surge como una manera de establecer un vínculo con el lugar y la cultura mapuche, por lo mismo, la lengua y el rescate del mapuzungun se presenta como la posibilidad de arraigarse a la tradición, a la oralidad y a la ancestralidad.

Existe una basta bibliografía sobre la poesía mapuche actual, que parte desde la academia chilena en la década de los ochenta con los aportes de Iván Carrasco. Sin embargo, los escritores del Puelmapu han sido invisibilizados en los estudios literarios trasandinos, así como ha sido también invisibilizada la cultura mapuche en general. Por lo mismo, los textos literarios y las distintas manifestaciones culturales abordan un coralidad de voces, como plantea Maribel Mora (2017) "los relatos y personajes que forman parte de la historia borrada, negada, tachada, en un gesto que busca aportar a la restitución del derecho a conocer la verdad histórica” (p. 176). 


\section{Elvira Rodríguez}

La invisibilización mencionada anteriormente se percibe también en las mismas publicaciones: escritores como Rubén Curricoy, Viviana Ayilef y Aylin Ñanchucheo han publicado a través de sellos independientes, auto gestionadas o en compilaciones de editoriales chilenas. Por lo mismo, la constitución de una poesía mapuche trasandina, así como reconocemos la poesía mapuche en el territorio chileno, ha sido un trabajo lento que apunta a la restitución de la memoria, en un proceso reivindicativo de lo mapuche desde distintas expresiones artísticas, como la música y la poesía.

Si comparamos la situación de los escritores mapuches, podemos entender que no es un hecho aislado, sino que se corresponde con cómo se ha construido el sujeto argentino, pues a nivel cultural y político, incluso biológico, se ejerce una borradura étnica. Recordemos que para efectos históricos se ha instaurado un discurso que apunta al carácter extranjero del pueblo mapuche, indicándolo como un invasor en el territorio y no como un pueblo originario (Radovich, 2013). Carlos Martínez Sarasola (2013) señala sobre este mismo tema que a la hora de conformar el perfil cultural argentino se piensa a partir de la gran migración europea que llega al país a mediados del siglo XIX, sin considerar la primera matriz cultural que son las comunidades indígenas que se encuentran y habitan el territorio mucho antes de la conquista española. Por lo tanto, la poesía mapuche argentina recoge la experiencia de los relatos faltantes, las historias borradas y se desarrolla en un contexto de producción escritural distinta a la que se desarrolla en el Wallmapu.

La obra de Liliana Ancalao -así como la de los distintos poetas que adscriben como mapuches- se inscribe en un contexto diferenciado de producción y recepción en relación a las letras argentinas. Su poemario Mujeres a la intemperiel Pu zomo wekentu mew (Suri Porfiado, 2010) reeditado en Resuello/ Neyen (Marisma, 2018) recupera la experiencia comunitaria de las mujeres. Desde el margen, las protagonistas dan sentido a la experiencia femenina en la ciudad en donde se establecen relaciones entre la escritura y la naturaleza, es decir, se desarrolla una poética vinculada al territorio. Hay un elemento que caracteriza la poesía de Liliana Ancalao que tiene que ver con el desplazamiento de las zonas rurales a la ciudad producto de las migraciones forzadas. En el contexto argentino, la reducción y empobrecimiento territorial, sumado a la explotación de los recursos naturales e hidrocarburos obliga la diáspora a los espacios urbanos, lo que se traduce en términos culturales en la incorporación de lo mapuche dentro del proyecto nacional, dejando de lado la diferencia y especificidad étnica, para formar parte del pueblo argentino. Lo que se conoce como argentinidad incorpora lo indígena como un número, pues como señala Andrea Szulc (2004): "- una vez neutralizados militarmente - quedan incorporados como «potenciales ciudadanos», aunque sin pleno acceso a los derechos de los cuales goza al resto de la población” (p. 168). En ese proceso de incorporación se mantiene el sesgo y se invisibiliza esta raíz cultural. El mapuche queda despojado o al margen de derechos y de la historia.

En este desplazamiento, el territorio -tan lejano a la ciudad del presente- se trae a la memoria a través de la escritura de Liliana Ancalao. Este se recuerda en los poemas, se revive y se reescribe como el lugar de la memoria. Por lo tanto, la experiencia femenina está cruzada por la distancia con la Mapu y por este nuevo escenario que le toca habitar, poblar y conseguir dominar. El objetivo del presente artículo es constatar cómo en el poemario Mujeres a la intemperie/ Pu 


\section{Elvira Rodríguez}

zomo wekuntu mew, reeditado como Resuello/ Neyen se establece un vínculo entre la experiencia femenina y la recuperación del territorio. Veremos que en los poemas seleccionados se desarrolla una relación directa entre el cuerpo y el lugar, dejando de manifiesto los distintos procesos históricos que se van grabando en la escritura de la autora. Para esto revisaremos cómo el lugar, aquella construcción que se da desde las letras, es fundamental para mostrar la historia faltante y los cuerpos que han sido olvidados en los relatos maestros. Veremos que estos lazos se restablecen incluso con la incorporación del mapudungun en los poemas. La poesía de Ancalao reúne la experiencia comunitaria de las mujeres que han sido borradas de la historia. Su historia, que es la historia de toda una generación, queda plasmada en sus poemas incorporándose a la multiplicidad de voces mapuche que han utilizado la escritura como forma de resistir a los relatos históricos monoculturales.

\section{Dicotomía ruralidad-ciudad: la escritura como recuperación territorial}

La dicotomía ruralidad-ciudad aparece en los versos que abren este artículo, en donde el mameluco se presenta como una alegoría del mundo laboral que marca a la generación de los padres que debieron dejar el territorio, pues como señala Ancalao (2018) son ellos quienes "Debieron dejar el campo porque no era suficiente para dar sustento a todos, cambiar el ciclo de la siembra y el ciclo de las pariciones, por un empleo, un salario, horarios y patrones" (p. 51). En los versos vemos cómo se escribe desde este nuevo espacio que se habita, pues el mameluco representa aquello que trae consigo la migración: las jornadas laborales, la pobreza, la mano de obra barata y la explotación de los recursos. Es el paso de una forma de vida a la otra, marcada por la explotación de la naturaleza y la migración desde la pampa a la ciudad, dejando el territorio de origen y las relaciones que se establecen en y con él, como señala la poeta: "Nacimos en el tiempo de la desmemoria. Fuimos niños y adolescentes sin memoria" (Ancalao, 2018, p. 54).

Para entender la relación que se establece entre la poesía y el territorio, trabajaremos el concepto de 'lugar' a partir de lo propuesto por Lawrence Buell (2005). Para la ecocrítica el lugar debiese ser también una categoría de análisis (Flys et al, 2010) y si bien nuestro estudio no se inscribe de manera exclusiva en aquella escuela, es imposible descartar la obra de su contexto político y ecológico, pues en este caso vemos además que se establece una relación entre las protagonistas de los poemas y su entorno. Sin embargo, no nos centraremos en una lectura exclusivamente ecofeminista, lo que nos interesa es la relación que se establece con el lugar, pues vemos en esta poética situada una forma de restitución. Por lo anterior, nos resulta fundamental la diferencia que plantea Buell entre lugar/ place y espacio/ space, en tanto el primero responde a la construcción que establecen los sujetos con su entorno y la naturaleza. El lugar, para el autor, aborda lo relativo a los afectos, a la materialidad ambiental y la crítica social. Es una construcción afectiva, política y de memoria. A diferencia de lo que se entenderá por espacio/ place, que se referirá a la delimitación geográfica y política. Buell señala: "la historia del mundo es una historia de espacios que devienen lugares. En el comienzo la tierra era un espacio sin forma. Luego, por la 


\section{Elvira Rodríguez}

habitabilidad se crearon los lugares"3 (nuestra traducción libre, Buell, 2005, pp. 63-64). En el caso de la poesía de Ancalao, el territorio está en un constante proceso de construcción: de espacio a lugar, recuperando a través de la escritura estas borraduras históricas que mencionamos antes.

Si reflexionamos sobre la relación que se establece entre lugar y espacio, y lo trasladamos a la idea de tierra/ territorio (Rodríguez, 2018) es posible establecer un quiebre tras la colonización europea en el continente. Niall Binns (2004), en su texto ¿Callejón sin salida? La crisis ecológica en la poesía hispanoamericana, señala lo siguiente:

La modernidad dio alas a los sueños de poder del ser humano, convirtiéndolo en un auténtico depredador de su entorno. Frente a la visión tradicional (tanto en la Europa premoderna como en la América precolombina) de un universo orgánico y animado, de una Tierra que el hombre veneraba y respetaba, surgió el pensamiento radicalmente desacralizador de Francis Bacon. La naturaleza dejaba de ser un ente sagrado y mágico: ahora sería puesta al servicio del capitalismo naciente, estudiada, "sometida a interrogatorio" en busca de sus secretos y dominada. (p. 10)

Este carácter sagrado no sólo se pierde con la llegada de occidente, se mantiene y se consolida con la instauración de los Estados nacionales modernos, que en sus breves historias tratan de establecer el orden y el dominio del territorio. Ese dominio implica la privatización y/o explotación de los recursos naturales. En ese contexto, el territorio mapuche -que no conoce fronteras nacionales- se reduce, empobrece y destruye a partir de la falsa idea de desarrollo. Si en un comienzo la idea de modernidad se instauró como el vehículo para sacar a los pueblos de la 'barbarie', posteriormente fue para sacarlos de la pobreza. Y en ese proceso, la naturaleza pierde su estatuto sacro que, lejos de revertirse con el tiempo, se intensifica con el paso de los años. Este proceso, además, se transforma en una herramienta que, desde los Estados nacionales, sirve para la unificación de un modelo político y económico.

Frente al embate modernizador bajo la premisa de la colonización, escribir sobre la naturaleza funciona como un ejercicio decolonial, en tanto la relación que se establece entre los Estados nacionales y los grupos originarios no dista mucho de aquella que se estableció en las colonias en relación a la construcción de una identidad territorial étnica (Parekh, 2000). Si pensamos, además, desde la justicia medioambiental que incluye los factores sociales (Flys, 2010), vemos que en aras del progreso los Estados están dispuestos a pagar ciertos costos, pero ¿qué costos?, ¿quién los asume? La experiencia nos ha demostrado que las zonas de sacrificio también son las zonas más pobres del país. Por lo tanto, escribir en este contexto, además de ser un acto medioambientalista de denuncia, es también una forma de oponerse a un modelo económico y a una forma de gobernar. Sobre esto mismo, Binns (2004) tiene una postura similar, pues para el autor: "la crisis ecológica es inseparable a la crisis étnica y la historia latinoamericana de la resistencia contra la conquista, la colonia y el neoimperialismo económico ha sido siempre, en el fondo, una larga lucha contra el etnocidio y el ecocidio" (p. 15). Por lo tanto, si desvinculamos la

\footnotetext{
3 "World history is a history of space becoming place. In the beginning earth was a space without form. Then through inhabitance places were created" (Buell, 2005, pp. 63-64).
} 


\section{Elvira Rodríguez}

crisis ecológica de los movimientos sociales, estamos dejando de lado la lucha por la recuperación del territorio.

Es en este contexto que ubicamos la obra de Liliana Ancalao, pues en su escritura el territorio juega un rol fundamental, ya que a través de él la autora va escribiendo un correlato de la historia mapuche: la historia de los desmemoriados y de los desterrados que se ven obligados a dejar el territorio producto de la usurpación de las tierras por parte del Estado, tierras que a su vez, están siendo explotadas. Es a través de la escritura desde el cuerpo y desde el territorio que Ancalao (2018) va grabando los distintos procesos históricos que van desde los primeros días — “mi mamá fue una niña en Cushamen/ andaba en alpargatas por la nieve" (p. 11) - hasta llegar al presente, marcada por la migración: "en la ciudad el frío/ nos raspa las escamas/ nos punza en la nuca” (p. 17).

\section{Territorio, lengua y cuerpo: la escritura como amparo}

Antes de comenzar con el análisis de los poemas, resulta fundamental detenerse en dos aspectos fundamentales de la obra de Ancalao. En un primer lugar, es importante destacar que la autora desde la década de los 80 en adelante ha tenido una participación en la esfera pública. Sus textos han sido publicados de manera ininterrumpida y es probablemente una de las pocas escritoras mapuche de Argentina que ha publicado sus textos en editoriales. El segundo aspecto fundamental de su obra es el carácter bilingüe. El territorio y el cuerpo se manifiestan en el poemario a través de la lengua, que entenderemos como la primera forma de resistencia en relación al Estado monolingüe y que, a la vez, funciona como la primera visibilización del territorio en el texto, entendiendo esto a partir de la traducción del mapudungun como "la lengua de la tierra". Sobre esto, Rodrigo Rojas (2009) reflexiona en torno a la función que tiene este ejercicio en la materialidad del libro, a partir de aquello nos preguntamos: ¿para qué escribir en ambos registros?, ¿quién es el lector de aquellos textos en mapudungun? Liliana Ancalao (2018) pareciera responder a estas preguntas en su ensayo "El idioma silenciado", pues para ella: "El mapudungun es el idioma de recuperación del orgullo y de la reconstrucción de la memoria” (p. 69). La escritora declara pensar y escribir en español, la impuesta lengua materna en la que ha construido su pensamiento, para traducirse después, pues ella afirma que: 'Encontré en la poesía en 'castilla' la posibilidad de expresar algo de la profundidad que me inundaba. Y la nostalgia de dios, es decir, de una cosmovisión, me llevó por el camino a recuperar su idioma" (p. 68).

La lengua se transforma en un medio para visibilizar esa cosmovisión faltante. Más allá de que exista un lector de aquellos textos, es la posibilidad de volver a ese espacio cultural que se construye a través del mapudungun, a ese espacio bilengual, pues como plantea Rodrigo Rojas (2009), el idioma más que una lengua, es un modo de vivir, pues: "es algo que trasciende al sonido, la sintaxis y al léxico; está más allá de que se tenga - o no - dos idiomas; trasciende la ley que instala temor y vergüenza a la hora de expresarse entre aquellos que no dominan la lengua hegemónica" (p. 23). El texto en mapudungun deja en manifiesto la existencia de la cultura otra, además, evidencia la posibilidad de pensarse en la propia palabra. En este gesto encontramos un acto de autonomía y desprendimiento epistémico sin siquiera leer los textos, pues este tipo de 


\section{Elvira Rodríguez}

registro bilingüe le permite a la autora revitalizar la lengua, recuperar la memoria y plasmarla en un texto, equiparar la lengua mapuche a la lengua española y, de manera significativa, mostrarla en el lugar principal de la página.

El ejercicio de escribir en mapudungun no es ingenuo, ni menos ha sido un camino fácil, pues la pérdida de la lengua tras la escolaridad forzada implicó llevar el idioma a la esfera más privada, haciendo difícil su traspaso generacional. A ello se suma la migración a las zonas urbanas, en donde la lengua funcionaba como un estigma, como lo señala Ancalao (2018), para quien su lengua se tradujo en "el idioma para expresar el dolor, el idioma del desgarro cuando el reparto de hombres, mujeres y niños como esclavos" (p. 66). Sobre esta misma relación con la lengua, Adriana Paredes Pinda (2005) se refiere a la escritura, pero sobre todo a la lengua castellana en relación a la fascinación que esta le produce. La poeta en el prólogo de su poemario $\ddot{U} i$ señala la violencia con la que se impone el idioma: "Fue la lengua castellana que nos ultrajó en primer lugar y en el último (la lengua y el pensamiento), pero no solo ella por supuesto, la lengua hispana nos ha violentado, lo confieso, nos ha socavado" (p. 9). Es en esta perdición, sin embargo, que también ocurre una metáfora interesante, pues la lengua castellana conquista, amorosamente, a la poeta, y si bien es una usurpadora, en esta violencia aparece el amor: "ha perdido, sin retorno tal vez, me ha mordido los pensamientos y yo "pecadora", pobre de mí, me ha enamorado de la lengua castellana meretriz" (p. 9). Por lo tanto, el ejercicio de escribir, ya sea en mapudungun o con la grafía del conquistador, es el primer gesto de resistencia y de recuperación del territorio. Para la Ancalao (2018), perder la lengua materna es el primer paso para perder la memoria, por lo mismo, recuperar el idioma y plasmarlo a través de la escritura es una forma de restituirla, como ella misma señala: "La función de la poesía como actividad actual del pueblo originario mapuche es aportar a la tarea colectiva de devolver la transparencia al territorio. Un territorio de tiempos y espacios reconstruidos desde la memoria y la militancia" (p. 58). La poesía permite recuperar este espacio cultural propio y el texto se transforma en un espacio político en donde se pone en tensión la identidad, las relaciones entre los Estados y el territorio, y se interpela también el sistema monolingüe impuesto.

El poemario Mujeres a la intemperie / Pu zomo wekuntu mew está dividido en cuatro largos poemas: "El frio/ Wutre", "La lluvia/ Mawün", "El viento/ Rürüf" y "Cuando me muera/ Feichi Lali". Estos textos son un tejido entre la experiencia personal y la memoria colectiva de las mujeres. Esta experiencia coral se reafirma en cada poema a través de los subtítulos: "Las mujeres y el frío", "Las mujeres y la lluvia" y "Las mujeres y el viento". Las aprendices de machi son las protagonistas y las encargadas de traspasar la oralidad. Sin embargo, tal como lo señala el título, están en la intemperie, sin techo y al descubierto, un juego de palabras entre la idea de la inclemencia y la desprotección, y es que su relato contiene aquello, contiene la voz de esas mujeres invisibilizadas en los relatos maestros, las que conservan la tradición viva y luchan contra el olvido, en una suerte de orfandad en donde ellas son las que mantendrán viva la llama de la memoria: "Las mujeres aprendemos/tarde/ que hay un tiempo en la vida/ en que sin intención/ vamos dejando huella de incendio/ por el barrio" (Ancalao, 2018, p. 15). 


\section{Elvira Rodríguez}

Las mujeres que protagonizan los poemas de Ancalao transitan entre dos planos. Por una parte, son herederas y portavoces de toda una tradición; por otro, circulan en el cotidiano, calentando la pava o esperando el transporte público. Son las desplazadas del territorio que están en las ciudades buscando su lugar. Esa dualidad las caracteriza y define y queda manifestada en los poemas:

el viento siempre vuelve

pero esta ciudad no se acostumbra

anda

cada vez

desaforado por las calles

a brochazos de tierra

borrándonos los pasos

(...)

como un tremendo viento

dicen que fue el malón

un torbellino en contra de los días

$\mathrm{y}$ eso que los antiguos eran duros

como rocas

firmes

ahí quedó su sangre

desparramada

me decías abuela

$\mathrm{y}$ tu recuerdo es el lago

al que me asomo

para sorber un trago. (Ancalao, 2018, pp. 29-33)

En los versos anteriores se entrelazan la historia presente con los relatos antiguos, como la venida del malón y la migración a la ciudad. Hay un desplazamiento geográfico que también está marcado por los tiempos: aquel territorio que se recuerda en la memoria versus la ciudad que se habita desde el presente. El lugar desde donde se está contando, en contraposición al lugar que se debería estar habitando. El viento vincula ambos planos: funciona como un elemento continuo entre ambos espacios y temporalidades. Es un soplo que da vida, pero también es una potencia aniquiladora que barre con la memoria y la tierra, que arrasó con los más fuertes y que amenaza en el presente con desvencijar las casas, por lo tanto, es tarea de las mujeres el resistir, esperar que este pase.

En el poema citado las mujeres son las guardianas de la memoria, la experiencia femenina está directamente vinculada con la tradición, pues funcionan en una doble temporalidad entre lo cotidiano y la espiritualidad, entre el presente y la preservación de la historia: "los olores/ la ropa/ se desafina la casa/ la memoria se astilla/ y hay que poner la pava" (Ancalao, 2018, p. 31). El día a día, las labores domésticas y la resistencia cultural se unen en esta experiencia coral.

Sobre la memoria, es a través de la figura materna que se reproduce y se mantiene la cultura, la transmisión de la lengua. Esto se representa en el poema a través de la figura de la abuela: "y tu 


\section{Elvira Rodríguez}

recuerdo es el lago/ al que me asomo/ a sorber un trago" (Ancalao, 2018, p. 33), pues la hablante primero tiene que acceder a la memoria, debe apropiarse de estos recuerdos que no son suyos, pero que sí le pertenecen, pues son parte de su linaje. Los recuerdos son del pasado, pero acordarse no es sólo traer un recuerdo a la memoria, es buscar y hacer algo desde el presente. Si la memoria se refiere a lo que un día fue, sería labor de las hablantes reconstruir aquella historia que no les perteneció. En el caso del poema, es a través de las mujeres que se activan los recuerdos en función de un pasado que les resulta lejano, y en esta activación, en la rememoración, se obtiene un nuevo significado.

Sobre el territorio, en "Las mujeres y el frío" Ancalao (2018) contrasta el espacio urbano que le toca vivir desde el presente con el Cushamen que habitó su madre:

yo al frío lo aprendí de niña en guardapolvo

estaba oscuro

el rambler clasic de mi viejo no arrancaba

había que irse caminando hasta la escuela

cruzábamos el tiempo

los colmillos atravesándonos

la poca carne

yo era unas rodillas que dolían

decíamos qué frío

para mirar el vapor de las palabras

y estar acompañados. (p. 11)

$\mathrm{Al}$ igual que en el poema anterior, es a través de la experiencia comunitaria de las mujeres que se desarrolla la acción del poema. Esta experiencia está marcada por el desplazamiento que se manifiesta en el contraste entre las generaciones: la experiencia de la madre en contraposición a la experiencia de la hablante, pues en el poema hay dos lugares: el primero, es el que habita en la memoria, en la infancia de la poeta en donde recuerda a su madre y su vida en Cushamen. El segundo es el presente desde el cual es evocado y funciona como una vertiente, un origen, que a su vez es comparado con el espacio que se rememora. Es un lugar que despierta tiempos pasados. El frío funciona como el canalizador de ambos planos, el frío de la infancia/ memoria no se compara con el del barrio/ presente. Este une las experiencias del poema y cambia según el lugar, como un personaje vivo dentro del poema que pareciera guardar un carácter sagrado, pues es azul, color que en sí reúne la espiritualidad mapuche. Sobre la figura de la madre, esta se presenta como el calor que guerrea contra la inclemencia. Este calor no solamente combate esta fragmentación que marca los distintos espacios, también permite la sobrevivencia, que crezcan las ramas y los brotes: "mi mamá nos abrigaba / ella es como un adentro (...) y defienden a los chicos del invierno/ y a veces sale el sol y ellas tapando/ porque los brazos se les van en vicio" (Ancalao, 2018, p. 13). Hay una experiencia que surge producto de esta presencia que está asociada a la pertenencia de la memoria: la figura de la madre permite a la hablante germinar como semilla que se seguirá reproduciendo. 


\section{Elvira Rodríguez}

Sobre el territorio, encontramos la ciudad en contraposición a la pampa, a la memoria, al recuerdo de la madre caminando en un entorno natural tan disímil al barrio. Sin embargo, la escritora no niega la posibilidad de vida en este nuevo espacio. Como dice la misma autora: "La ciudad fue una posibilidad laboral y una posibilidad de estudio para los brotes. Se vinieron nuestros padres monolingües, sin nguillatun, sin mapuzungun. A cambiar el ciclo natural del tiempo por horarios de trabajo y calendario escolar" (Ancalao, 2018, p. 68) y aquello se percibe en el poema en tanto la figura femenina alberga un fuego que va dejando una huella, una marca. La figura de la madre es la que va perpetuando la memoria y cuidando a los brotes, en una analogía de la naturaleza y la descendencia:

hay que abrigar a los hijos

el pecho

la espalda

los pies y las orejas

dicen así

y les crecen las ramas y las hojas (Ancalao, 2018, p. 13).

En los poemas que hemos seleccionado, la naturaleza se presenta como un reflejo de los hablantes, como una proyección de la mujer dentro de los textos. Vemos cómo las emociones, reacciones y acciones son reflejadas en la forma en que se describe el espacio natural. En este poemario compuesto a partir de la memoria de la escritora y de las mujeres a las que representa, se entrama un discurso en donde se entrelazan las historias personales y familiares, explorando los distintos rincones de la memoria y el presente. En ese contexto, la naturaleza funciona como esta matriz que contiene a la hablante. La escritora, además, hace un reciclaje de la sonoridad del mapudungun y los ruidos que abundan en el territorio:

yo a las palabras las pienso

y las rescato del moho que me enturbia

cada vez puedo salvar menos

y las protejo

son la leña prendida de atahualpa

que quisiera entregar a esas mujeres

las derramadas las que atajan sus pájaros. (Ancalao, 2018, p. 25)

El trinar de los pájaros se mimetiza con la voz de las mujeres y el sonido del viento; el resuello se confunde con el cabalgar de los caballos; el frío es una metáfora del desarraigo y la tristeza. En el poema "Las mujeres y la lluvia" la experiencia está vinculada con la naturaleza, como una proyección, como una extrapolación de lo femenino en el mundo natural. La experiencia femenina está marcada por la naturaleza, por ejemplo, en la idea de la luna y la fertilidad:

cuando niñas vamos sueltas por el patio

y el sol nos persigue de a caballo

pero la luna implacable nos va dejando sus mareas 


\section{Elvira Rodríguez}

hasta que nos desvela

y esa noche encontramos

un cántaro

en lugar de la cintura (Ancalao, 2018, p. 19).

El cuerpo femenino, así como las mareas, cambian con los ciclos lunares transformando a las niñas, las convierte en mujeres, en cántaros, pero ¿qué cobijan estos cántaros? ¿la posibilidad de dar vida o ser la vida misma? Las mujeres son vasijas de la memoria que va gestándose, que se ha heredado y que va brotando.

En estos poemas, el cuerpo opera como un pretexto literario, en tanto funciona a la vez como un elemento simbólico dentro de los poemas que rescata la memoria y nos direcciona a la visualización de esta corporalidad, que parece silenciada en este espacio cultural hegemónico argentino y que es capaz de reproducirse. En la cosmovisión mapuche, en lo femenino reside en el norte, con el frío y la noche, elementos que se incorporan en los poemas. En este espacio habitan las fuerzas del mal, las potencias destructoras, aquello que amenaza el orden. Sin embargo, en los poemas seleccionados se revierte este orden y las mujeres cumplen con un rol fundamental, en tanto son dadoras de vidas, son machis, portadoras de la memoria, la palabra y la tradición (Rodríguez, 2014).

En el caso del cuerpo menstruante, este es sinónimo de vida, pero también de debilidad y destrucción:

La sangre cíclica y femenina está asociada a una fuerza de destrucción y de atracción. En el primer caso, se piensa que si una mujer pisa tierra recién sembrada los frutos podrán marchitarse; en el segundo, si se le administra a un hombre una pócima de agua con flujo menstrual éste se enamorará hasta llegar a someterse a la dueña de la poción. Como se aprecia, debilidad y poder operan simultáneamente en el cuerpo femenino. (Montecino, 1984, p. 16)

En el poema se invierte esta situación y el cuerpo, transformado en cántaro por la luna, es una potencia de vida que va a llenar, a modo de lluvia, los territorios. Este elemento simbólico funciona como una alegoría de la intensidad de las mujeres que las acompaña desde el rocío, desde que despunta la mañana y que se cuela en el cotidiano. La llovizna se desprende del cuerpo y desde ahí se desatan las emociones que bañan y envuelven al resto. Estas emociones son explosivas y se asemejan a los distintos estados también de la lluvia. Esta explosión también está vinculada con la experiencia ancestral, con cai cai, que inunda las tierras y va borrando los trazos. Sin embargo, esta presencia devastadora se revierte hacia el final del poema, pues la tierra mojada se asocia al goce de la hablante: "huele tan bien la tierra después del aguacero" (Ancalao, 2018, p. 27).

En el poemario el desplazamiento a las zonas urbanas se presenta a través de la relación que se establece con la imagen de Cushamen, territorio que se construye desde la memoria, y la ciudad que se habita desde el presente. Esa construcción se realiza desde la experiencia femenina, en tanto son las que caminan en la intemperie, pero dando cobijo a los brotes. 


\section{Elvira Rodríguez}

\section{Conclusión}

En los poemas seleccionados podemos rastrear un discurso que hace frente a lo que Álvaro Bello llama "la maquinaria civilizatoria, la que desde fines del siglo pasado se planteaba el cómo terminar con el "problema mapuche"" (2004, p. 14). En los poemas encontramos un discurso que apunta a la diferenciación, pero sobre todo a una discursividad propia en donde la mayor parte de las veces encontramos un pensamiento decolonialista que incluye la cosmovisión, la tradición, la historia y el territorio, que irrumpe el Estado monolingüe argentino. Sobre esto, Liliana Ancalao (2018) señala: "La conciencia de ser parte de un pueblo nos hace ser responsables del resguardo de una cultura, porque la pérdida de la memoria, el olvido (...) acecha a los pueblos originarios hoy: Memoria y conocimientos son sinónimos" (p. 62).

La escritora hace un reciclaje de la sonoridad del mapudungun, de los ruidos de la pampa, el viento que se cuela en la ciudad, la lluvia y los pájaros. Rescata la propia lengua y, tal como señalamos al comienzo, el gesto de escribir en el idioma de la tierra es el primer gesto de autonomía. Se establece un vínculo entre la experiencia de ser mujer y la recuperación del territorio. El lugar - tanto cuerpo como territorio- descrito en los poemas permite restituir la experiencia femenina, poner en tensión los discursos totalizadores identitarios.

La poeta, para situarse en aquella identidad, primero debe echar mano a su pasado, recuperar su historia, y es por medio de la experiencia natural que lo consigue, recuperando la palabra que brota del kultrün, dotándola de una experiencia y voz particular que está cruzada por la experiencia de ser mujer y el lugar. Los elementos naturales funcionan como una proyección, maximizando las sensaciones de esta nueva mujer que se construye en la ciudad. No es una relación esencialista entre naturaleza y mujer, sino que una construcción a partir de los mismos procesos históricos que hemos mencionado anteriormente, que permiten que el territorio se reconstruya junto con la identidad.

En la escritura de Ancalao se establece una relación entre la mujer y la proyección de la naturaleza, que le permite constituirse en el nuevo escenario que habita. Describe y escribe la tierra en función de esta nueva realidad, rescata las raíces para conocer y sembrar el presente. La naturaleza constituye a la sujeta, es una dependencia para entenderse en este proceso, en esta constante escritura, lo que nos da un guiño con el ecofeminismo como posible forma de lectura de esta obra.

Los desmemoriados, como los llama Ancalao (2018), son los hijos y los nietos que llegaron a poblar las ciudades a mediados del siglo XX y que siguen deambulando por las calles tratando de encontrar su espacio en las urbes. La poesía de Ancalao se presenta como este ejercicio constante de hacer memoria, visibilizar a los brotes que andan deambulando por las ciudades. Es devolverles la lengua y el territorio, recuperar el mapudungun y transformarlo desde la lengua del desarraigo a la lengua de la memoria. Ejercicio que vemos en autoras como Adriana Paredes Pinda y Graciela Huinao en su poemario Walinto.

Restituir la dignidad no sólo ancestral, también pensar la situación del mapuche en la actualidad, reconociendo en el lenguaje, otrora herramienta colonizadora, un recurso para la sobrevivencia. La literatura de Ancalao se suma a un corpus de escritores que transgreden el 


\section{Elvira Rodríguez}

espacio monolingüe, llevando el territorio y la lengua a la ciudad, rescatando la singularidad de sus raíces para que otros habiten también esos lugares.

Todo lo anterior es una pequeña muestra de cómo en la literatura también se desarrolla un discurso contrahegemónico, en donde se rescata el lugar en relación a una visión totalizadora y desacralizada que tienen los Estados, sobre todo en el argentino, que se ha encargado de blanquear sus raíces. En un primer lugar, vemos el rescate de las voces que han sido silenciadas, nos enfrentamos a la historia otra no contemplada en los proyectos nacionales.

La poesía pone en tensión los discursos oficiales y le permite a la escritora colarse en la discusión política, resignificando las relaciones que establecen entre los estados monoculturales y los grupos subalternos. Permitiendo, además, resistir al paso del tiempo y darles a los nuevos brotes un lugar de encuentro.

\section{Referencias:}

Ancalao, L. (2009). Mujeres a la intemperie. Pu zomo wekuntu mew. Buenos aires, Argentina: El suri porfiado.

Ancalao, L. (2018). Resuello. Neyen. Madrid: Marisma.

Araya, J. (2016). Aproximaciones al estudio ecocrítico de la literatura chilena. Logos: Revista de Lingüística, Filosofía y Literatura, 26(2),278-285. https://doi.org/10.15443/RL26021

Bello, A. (2004). Intelectuales indígenas y universidad en Chile: conocimiento, diferencia y poder entre los mapuche. En R. Austin. (Ed.). Intelectuales y educación superior en Chile: de la Independencia a la transición democrática 1810-2001. (pp. 97- 132). Santiago de Chile: Editorial CESOC.

Binns, N. (2004). ¿Callejón sin salida? La crisis ecológica en la poesía hispanoamericana. Zaragoza: Prensas de la Universidad de Zaragoza.

Buell, L. (2005). The Future of Environmental Criticism: Environmental Crisis and Literary Imagination. Malden: Blackwell Publishing.

Casals, A. (2014). Fundamentos para la lectura ecocrítica en Chile. (Tesis doctoral). Pontificia Universidad Católica de Chile.

Flys, C. (2010). Literatura, crítica y justicia medioambiental. En C. Flys, J. M. Marreno y J. Barella. (Comp.), Ecocríticas. Literatura y medio ambiente. (pp. 85- 120) Madrid: Iberoamericana.

Huinao, G. (2009). Walinto. Santiago de Chile: Editorial Cuarto Propio.

Martínez Sarasola, C. (2013) . Nuestros indios los paisanos. Buenos Aires: Del nuevo extremo.

Mellado, S. (2014). Lenguas Kuñifal: Pasajes entre el Mapuchezungun y el castellano en Elicura Chihuailaf, Liliana Ancalao y Adriana Paredes Pinda. Recial, 5-6, 1-18 https://doi.org/10.53971/2718.658x.v5.n5-6.9586

Montecino, S. (1984). Mujeres de la tierra. Santiago de Chile: CEM.

Mora Curriao, M. (2018). Muestra de poesía mapuche. Trazas poéticas sobre una cartografía indígena incesante. Anales de la Universidad de Chile, (13), 165-218. $\underline{10.5354 / 0717-8883.2018 .49003}$ 


\section{Elvira Rodríguez}

Moraga, F. (2011). Antesala inaugural en la escritura poética de mujeres mapuche durante las décadas de los años 70 y 80. En Moraga, F y Mora, M (Eds.) Kümedungun / Kümewirin. Antología poética de mujeres mapuche (Siglos XX - XXI). Santiago de Chile: LOM Editores.

Parekh, B. (2000). El etnocentrismo del discurso nacionalista. En A. Fernández. (Comp.), La invención de la nación. Lecturas de la identidad de Heder a Homi Bhabha. (pp. 91- 120) Buenos Aires: Ediciones Manantial SRL.

Paredes Pinda, A. (2005). Ül. Santiago de Chile: LOM Editores.

Rodríguez, E. (2014) El cuerpo como (pre) texto literario. Estudios Avanzados, 21 (jun), pp. 91110.

http://www.revistaidea.usach.cl/ojs/index.php/ideas/article/view/1593

Rodriguez, E. (2018). El lugar como espacio de denuncia y significación en el poemario Wafpule

Mülenymun (Aquí estamos) de Rubén Currico, English Studies in Latin America, 14. http://esla.letras.uc.cl/images/PDFS/ESLA-14/RODRIGUEZ.pdf

Radovich, J. (2013). Los mapuches y el estado neuquino: algunas características de la política indígena, Runa $\mathrm{n}^{\circ}$ XXXIV (1), 13 - 29. Recuperado de http://www.scielo.org.ar/pdf/runa/v34n1/v34n1a02.pdf

Rojas, R. (2009). La lengua escorada. la traducción como estrategia de resistencia en cuatro poetas mapuche. Santiago de Chile: Pehuén.

Stocco, M. (2017). La autotraducción en la poesía mapuche como territorio de tránsitos, tensiones y resistencias. Estudios filológicos, (59), 185-199. http://dx.doi.org/10.4067/S0071-17132017000100010

Stocco, M. (2018). Traful: una propuesta de estudio de la autotraducción en poesía mapuche, Literatura: teoría, historia, critica, vol. 20, (1), $39-61$. http://dx.doi.org/10.15446/lthc.v20n1.67276

Szulc, A. (2004). <Mapuche se es también en la waria (ciudad)>. Disputas en torno a lo rural y lo indígena en la Argentina, Política y sociedad, vol 41, (3), 167 - 180. Consultado de http://revistas.ucm.es/index.php/POSO/article/view/POSO0404330167A/23157 